NASA Contractor Report 187132

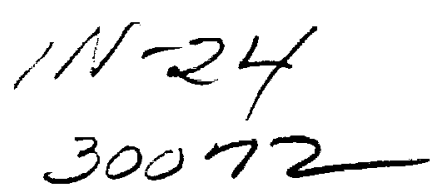

R33

\title{
Mechanical Behavior of a Continuous Fiber Reinforced Aluminum Matrix Composite Subjected to Transverse and Thermal Loading
}

S. Jansson and F.A. Leckie University of California Santa Barbara, California

June 1991

Prepared for

Lewis Research Center

Under Grant NAG3-894

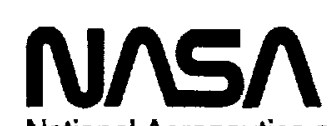

National Aeronautics and

Space Administration

(NASA-CR-197132) MECHANICAL BEHAVIOR OF A

NQ1-27243 CONTINUDUS FITER REINTORCEO ALUMINUM MATKIX

COMPUSITE SUDJECTED TO TRANSVERSE AND

THERMAL LOAOING Final Report (California 


\title{
Mechanical Behavior of a Continuous Fiber Reinforced Aluminum Matrix Composite Subjected to Transverse and Thermal Loading
}

\author{
by \\ S. Jansson and Frederick A. Leckie \\ Department of Mechanical and Environmental Engineering \\ University of California \\ Santa Barbara, CA 93106
}

\begin{abstract}
The transverse properties of an aluminum alloy metal matrix composite reinforced by continuous alumina fibers have been investigated. The composite is subjected to both mechanical and cyclic thermal loading. The results of an experimental program indicate that the shakedown concept of structural mechanics provides a means of describing the material behavior. When the loading conditions are within the shakedown region the material finally responds in an elastic manner after initial plastic response and for loading conditions outside the shakedown region the material exhibits a rapid incremental plastic strain accumulation.

The failure strain varies by an order of magnitude according to the operating conditions. Hence for high mechanical and low thermal loading the failure strain is small, for low mechanical and high thernial loading the failure strain is large.
\end{abstract}




\section{INTRODUCTION}

The potential for weight and strength advantages of components made of metal matrix composites is the consequence of the anisotropic properties of the composite. That advantage is diminished, or is even lost, for laminates with a less marked anisotropy. Consequently if full advantage is to be taken of the dominant strength characteristics then the fibers should be oriented in the direction of maximum stress transmission. In this circumstance the transverse properties of the composite are critical since there must be sufficient strength in the matrix to carry the secondary stresses applied in the transverse direction.

The transverse properties of a metal matrix composite consisting of an aluminum lithium alloy matrix reinforced with continuous alumina fibers are investigated in this study. An important characteristic of this material is the combination of a strong bond at the fiber-matrix interface and a ductile matrix. There is also a large mismatch in the coefficient of thermal expansion of the fiber and matrix so that fluctuations in operating temperature induce thermal stresses in the composite. It is the goal of this study to determine the behavior of the composite, when subjected to mechanical and thermal loading with special attention given to the transverse properties. The properties in the fiber direction are the subject of another study. As a result of this study it is possible to uescribe the behavior of the composite in terms of the shakedown concept used in structural mechanics, and it is also possible to develop a rather simple method for establishing the constitutive equations for use in structural calculations.

\section{EXPERIMENTAL PROGRAM}

The composite studied is Du Pont's FP/Al [Champion et. al., 1978], with continuous fibers in a unidirectional lay-up. The fiber volume fraction was determined to be $55 \%$. The FP fiber consists of $99 \%$ polycrystalline $\alpha$-alumina $\left(\mathrm{Al}_{2} \mathrm{O}_{3}\right)$ coated with silica that improves the strength of the fiber and aids the wetting by the molten metal. The fibers have a diameter of approximately $20 \mu \mathrm{m}$, a modulus of 345 to $380 \mathrm{GPa}$, a tensile strength of 1.9 
to $2.1 \mathrm{GPa}$ for $6.4 \mathrm{~mm}$ gauge length, and a fracture strain of $0.3-0.4 \%$. The matrix material is a $2 \mathrm{wt} \% \mathrm{Li}-\mathrm{Al}$ binary alloy. The lithium promotes the wetting of the alumina fibers that forms a strong matrix-fiber interface and it also raises the modulus and decreases the density of the aluminum. The composite is fabricated by preparing the FP fibers into tapes by using a fugitive binder and the tapes are subsequently laid up in a metal mold in the desired orientation. The binder is burned away and the mold is vacuum-infiltrated with the molten matrix. The composite was available in the form of a plate $150 \times 150 \times 12.5$ mm thick.

The specimen used in the test is shown in Fig. 1a. It has a relatively large radius at the transition from the gripping section to the reduced gauge section to provide a low stress concentration and a short gauge length for efficient use of the material. The specimen was loaded in a servo hydraulic machine and it was heated by means of induction coils (Fig. 1b) and the strain was measured with an extensometer with $3 / 8$ " gauge length. The temperature was measured by using three type $\mathrm{K}$ thermocouples mounted at the center and at the ends of the gauge section. The center thermocouple controlled the temperature while the top and bottom thermocouples were used to measure the variation of temperature along the length of the specimen. The variation of temperature with time and space is shown in Fig. 2 . The spacial temperature distribution is slightly different for the heating and cooling parts of the cycle. It was not possible to adjust the coil to have a uniform temperature distribution over the whole cycle. It was therefore adjusted to have a minimal spacial variation for both parts of the cycle. A computer was used to control the tests by generating command signals for load and temperature and to perform data acquisition.

The tests reported in this study involved a constant transverse stress in combination with cycles of temperature with cycle time $150 \mathrm{~s}$. Because of the limited availability of the composite only one specimen was used for each transverse stress level. The specimen was loaded and subjected to cyclic temperature and the ratcheting rate was measured when the steady state condition was reached. The cyclic temperature range was then increased and the next rate was measured on reaching the next steady state condition. The temperature and strain variations were continuously recorded and typical examples of readings are 
shown in Figs. 3, 4 and 5. The strain measured over a cycle of temperature is shown in Fig. 3, which indicates a small amount of hysteresis which it is due to the small spatial time-dependent nonuniformity of the temperature field in the specimen. The accumulation of strain in the direction of the applied stress are shown for low transverse stress in Fig. 4, and for a high stress in Fig. 5.

\section{EXPERIMENTAL OBSERVATIONS}

Transverse stress-strain curves at room temperature are shown in Fig. 6, from which a deviation from linearity is observed to occur at $75 \mathrm{MPa}$. The ultimate strength is 200 $\mathrm{MPa}$ and the strain to fracture is $0.8 \%$. The ultimate strength is about $50 \%$ higher than the ultimate matrix strength while the failure strain of $0.8 \%$ is only $3 \%$ of the $30 \%$ failure strain of the matrix [Sakui and Tamura, 1969].

Representative results for the constant transverse stress and cyclic temperature are given in Figs. 4 and 5 which indicate the results for a low and high transverse stress respectively. In both cases transient behavior is followed by a cyclic response for which there is an increment of strain after each cycle, i.e., ratcheting occurs in both examples. For the low transverse stress the transient portion is completed after one cycle (Fig. 4) whereas in the case of the high load the transient behavior continues for forty cycles before a steady state condition is reached (Fig. 5).

Similar tests were performed at different values of constant transverse stress and temperature cycle. In Fig. 7 , the steady state strain range $\Delta \varepsilon$ recorded over a cycle of temperature is plotted as a function of the temperature range $\Delta \mathrm{T}$ for different values of the transverse stress $\sigma_{\mathrm{T}}$. It may be inferred from this plot that the cyclic strain is independent of the level of the transverse stress and is linearly dependent on the temperature range $\Delta T$.

In Fig. 8 contours of constant values of $\mathrm{d} \varepsilon_{\mathrm{p}} / \mathrm{dN}$ are plotted, where $\varepsilon_{\mathrm{p}}$ is the plastic ratchet strain and $\mathrm{N}$ is the cycle number. There are combinations of $\sigma_{\mathrm{T}}$ and $\Delta \mathrm{T}$ for which no ratcheting occurs and after an initial (transient) response the cyclic behavior material is elastic. This condition is indicated in Fig. 8 as the shakedown condition. When the operating conditions exceed the shakedown condition ratcheting occurs at rates indicated in 
Fig. 8 and Fig. 9.

The contours of constant ratchet strain rate plotted in Fig. 8 are generally parallel to the shakedown surface. This observation suggests that the force $\beta$ which drives the ratchet strain rate is given by

$$
\beta=\left[\frac{\Delta \mathrm{T}}{\mathrm{T}_{\mathrm{s}}}+\frac{\sigma_{\mathrm{T}}}{\sigma_{\mathrm{s}}}-1\right]
$$

where $T_{S}$ and $\sigma_{S}$ are the ordinates defining the shakedown condition. The relationship of $\mathrm{d} \varepsilon_{\mathrm{p}} / \mathrm{dN}$ has the form

$$
\frac{d \varepsilon_{p}}{d N}=f(\beta)
$$

where $f(\beta)$ has the form given in Fig. 10. The relationship is exponentially dependent on $\beta$ and can be written as

$$
f(\beta)=\exp \left(9.5 \cdot 10^{2} \beta\right)-1
$$

The failure strain is dependent on the operating condition as indicated in Fig. 11. The failure strain was $0.8 \%$ for high stress and low thermal load whereas for low transverse stress of $30 \mathrm{MPa}$ the failure strain reaches $12 \%$. Microscopic observations of specimens subjected to low transverse loading and with large failure strains, Fig. 12a, showed distributed damage in the form of small cracks over the whole gauge section. The cracks are initiated from areas with poor matrix infiltration and locations with closely spaced fibers. The macroscopic fracture surface is wavy. A higher magnification view Fig. 12b, indicates a ductile fracture in the matrix with extremely oblong voids. It appears that the initial fracture is close to the fiber matrix interface on planes perpendicular to the loading direction and that the final fracture consists of a ductile fracture in the remaining matrix 
ligaments between the fibers.

The fracture for high transverse loading and small failure strain, Fig. 13a, is localized to one narrow band oriented approximately $45^{\circ}$ to the loading direction. The fracture surface, Fig. 13b, also indicates that the fracture is governed by a ductile matrix failure. However, the fracture does not approach the fiber matrix interface as for the low transverse loading and it appears as the whole load carrying capacity of the matrix has been lost at the same time. These observations suggest that the failure mechanism in the matrix is associated with void growth.

\section{COMPUTATIONAL STUDIES}

By using the theory of homogenization in conjunction with finite element procedures an attempt is made to determine the mechanics that governs the behavior of the composite in terms of the properties of the fiber and the matrix.

The present composite consists of long fibers in a unidirectional lay up that are randomly distributed in the transverse plane. In the model to be analyzed the fibers are assumed to be long parallel cylinders arranged in a hexagonal array, Fig. 14a. This periodical array has the mechanical properties with the closest symmetries to a composite with randomly distributed fibers. Both systems are transversely isotropic when the constituents are linear elastic but the hexagonal array has a weak deviation from transverse isotropy when the matrix exhibits a nonlinear stress strain relation [Jansson, 1990]. The deviation is most pronounced for a perfectly-plastic matrix. However, reasonable results can be expected if effective properties are calculated for loadings that do not permit slip planes unconstrained by the fibers.

The governing boundary value problem for the effective properties of the unit cell is two dimensional and has been solved with the Finite Element method by using ABACUS [1988]. A 10 node biquadratic quadrilate generalized plane strain element with reduced integration was used to avoid locking. The considered loading of the unit cell, Fig. 14a is symmetric with respect to the $y_{1}$ and $y_{2}$ axis. This implies that only an eighth of the 
indicated unit cell in Fig. 14a needs to be analyzed. The mesh shown in Fig. 14b is subject to the following in-plane boundary conditions for the tractions $T_{i}$ and displacements $u_{i}$ on the boundary S:

$$
\begin{aligned}
& T_{i}\left(\frac{\sqrt{3}}{2} b, \frac{b}{2}+c\right)=-T_{i}\left(\frac{\sqrt{3}}{2} b, \frac{b}{2}-c\right) \\
& u_{i}\left(\frac{\sqrt{3}}{2} b, \frac{b}{2}+c\right)=-u_{i}\left(\frac{\sqrt{3}}{2} b, \frac{b}{2}-c\right) \\
& u_{2}\left(y_{2}=0\right)=-u_{2}\left(y_{2}=b\right)=\text { const. } / \int_{y_{2}=0} T_{2} d S-\int_{y_{2}=b} T_{2} d S=0 \\
& u_{1}\left(y_{1}=0\right)=-\frac{\sqrt{3}}{2} b<\varepsilon_{1}> \\
& \frac{1}{b} \int_{y_{2}=0} T_{1} d S=-<\sigma_{1}>
\end{aligned}
$$

where $\left\langle\sigma_{1}\right\rangle$ is the average stress and $\left\langle\varepsilon_{1}\right\rangle$ is the average strain in the 1-direction. The generalized plane strain condition gives

$$
\varepsilon_{3}=\text { const. } / \int_{y_{3}=0} T_{3} \mathrm{~d} S=0
$$

A detailed description of the deviation of the boundary conditions for different loadings are given in Jansson [1990].

The elastic properties of fiber and matrix are not greatly affected by the history of processing and heat treatment of the composite so that it is possible to use data from the literature. However, the flow properties of the Al-Li matrix alloy are strongly dependent on histories of heat treatment and cold-working [Stark et al., 1981 and Sakui and Tamura, 1969]. Details of the processing of the composite and of any post heat treatments are not 
available. Hence, the exact state of the matrix is not known and it is not possible to extract the flow properties of the matrix from the literature. The only means of estimating the flow properties of the matrix of the composite is to select matrix properties so that the calculated response fits the experimental stress-strain curve for the composite. This procedure has been performed by Jansson [1990] who demonstrated that the matrix properties obtained from one stress state could be used to predict accurately stress-strain relationships for other loading states. The initial yield stress of the matrix was determined to be $94 \mathrm{MPa}$ with a hardening exponent $n=5$ (Fig. 6) for isotroptic hardening. The isotropic hardening cannot describe the matrix behavior when it is subjected to cyclic loading conditions and nonlinear kinematic hardening would be more appropriate. However, this option is not available in ABAQUS and the tests required to determine the cyclic properties of the matrix have not been performed for lack of material. The matrix was therefore modeled as an elastic perfectly-plastic material. In the calculations the fibers are assumed to be linear elastic and the matrix behavior is modelled with a small strain $J_{2}$ perfectly plastic theory using the properties given in Table 1. It is therefore not expected that the calculations can be used to provide accurate predictions but should be sufficiently reliable to provide insight into the material behavior.

The calculated transverse stress strain curve, Fig. 6, for an elastic perfectly plastic matrix agrees well with the experimental curve up to $\varepsilon=0.1 \%$. The calculated limit load is much lower than the measured because the matrix hardening has not been included. It can be noted that the increase in limit load is $30 \%$ for the perfectly plastic matrix. A substantial portion of the increase comes from the plane strain condition for the matrix in the fiber direction which is $2 / \sqrt{3}$ and the remainder represents constraint. The calculated cyclic thermal strain agree well with the measured values, Fig. 7.

The calculated response for constant stress and cyclic temperature, Figs. 15 and 16 exhibit the same features as the experiments, Figs. 4 and 5 with a short transition period for low transverse stress and a long transition period for high transverse stress.

In performing the elastic-plastic calculation it was possible to determine the shakedown boundary defined in Fig. 17. This has been expressed in terms of the 
dimensionless loadings $\mathrm{E} \Delta \alpha \Delta \mathrm{T} / \sigma_{\mathrm{y}}$ and $\sigma_{\mathrm{T}} / \sigma_{\mathrm{y}}$. It was found for an experimentally temperature dependent yield stress that the shakedown boundary is given by the result for a temperature independent yield stress to a good approximation by replacing the yield stress with the average yield stress for the temperature dependent case. The ratchet rates for different loading conditions fall one master curve, Fig. 18, when plotted as a function of $\Delta \varepsilon_{\mathrm{T}} \sigma / \sigma_{\mathrm{L}}$ where $\Delta \varepsilon_{\mathrm{T}}$ is the thermal strain increment outside the shakedown surface and $\sigma / \sigma_{\mathrm{L}}$ is the current transverse stress over the limit stress.

It was observed earlier that the failure strain was found to be strongly dependent on the transverse stress, Fig. 11. It is known that ductility is usually strongly dependent on the void growth factor $\sigma_{\mathrm{kk}} / \bar{\sigma}$ for ductile fracture. The highest void growth factor in the matrix is plotted in Fig. 19 versus accumulated transverse strain for different loadings. For transverse tension it increases from 3 at the initial linear elastic response to 6 at the observed fracture strain. Heating causes a negative hydrostatic pressure in the matrix. Transverse loading combined with thermal cycling causes a decrease in $\sigma_{\mathrm{kk}} / \bar{\sigma}$ during the heating after the initial transverse loading. During the subsequent cooling, the magnitude of $\sigma_{\mathrm{kk}} / \bar{\sigma}$ increases. The calculations indicate that the value of $\sigma_{\mathrm{kk}} / \bar{\sigma}$ decreases initially especially for low values of transverse stress. However, as strain is accumulated $\sigma_{\mathrm{kk}} / \bar{\sigma}$ increases and reaches a steady state condition with an increase of $\sigma_{\mathrm{kk}} / \bar{\sigma}$ for each cycle.

The computations indicate that the magnitude of the highest value in a cycle of $\sigma_{\mathrm{kk}} / \bar{\sigma}$ for a given accumulated transverse strain is strongly dependent on the magnitude of the transverse stress, Fig. 20, with low transverse stress requiring more strain than high transverse stress.

In Fig. 21 the equivalent plastic strain at the location with the highest value $\sigma_{\mathrm{kk}} / \bar{\sigma}$ is plotted against the accumulated transverse strain for different loadings. From this figure it can be deduced that the equivalent strain is linearly related to the transverse strain and is relatively independent of the transverse loading. 


\section{ANALYSIS OF THE EXPERIMENTAL AND COMPUTATIONAL STUDIES}

The experiments indicate that after transient response the material reaches a steady state condition. If the operating point lies within the shakedown condition the behavior of the material is elastic. When the shakedown condition is exceeded then steady state ratcheting occurs. The contours of constant ratcheting rates are found to be parallel to the surface defining the shakedown surface. Computational studies based on the assumption that the matrix is elastic-perfectly plastic also predicts shakedown behavior but the shape of the predicted shakedown surface is slightly convex while the experimental results fall on a straight line. However the computational predictions and experimental results are sufficiently close to confirm that the shakedown concept is valid. It is observed experimentally that the ratchet strain rate has the form

$$
\frac{d \varepsilon_{p}}{d N}=f(\beta)
$$

where

$$
\beta=\frac{\Delta T}{\Delta T_{s}}+\frac{\sigma_{T}}{\sigma_{s}}-1
$$

is proportional to the distance outside the shakedown surface and $f(\beta)$ is defined in Fig. 10. $\Delta T_{S}$ and $\sigma_{S}$ are the intersections defining the shakedown relationship, Fig. 8. The simplified analysis performed in the Appendix for the Tresca yield condition gives similar results and supports the possibility that equation (3) is valid. However, the computer calculations predict a more complex structure of the expression for the ratcheting rate with the form

$$
\frac{d \varepsilon_{p}}{d N}=\frac{\sigma}{\sigma_{L}} f(\beta)
$$

where $f(\beta)$ is equal to the thermal strain outside the shakedown condition and is then 
proportional to the distance outside the shakedown surface, Fig. 17, in the temperature direction. This is consistent with the model in the Appendix for the v. Mises yield condition.

The calculations and general results for shakedown conditions, cf. Ponter and Cocks [1982], indicate that the shakedown condition should intercept the stress axis at the limit stress. However, the experimental values intercept at a slightly lower stress (170 MPa) compared to the measured limit stress of $200 \mathrm{MPa}$. This discrepancy may be caused by the simple constitutive equations used in the calcualtions. The experiments intercept the temperature axis at $130^{\circ} \mathrm{C}$ and the calculations predict $110^{\circ} \mathrm{C}$. This is close in view of the uncertanty of CTE's of fiber and matrix and yield stress.

An upper bound on the rachet strain per cycle has been determined by Ponter and Cocks [1982]. The upper bound applies for loading conditions which just exceed the shakedown condition. The upper bound is given by

$$
\frac{\Delta \varepsilon_{p}}{\Delta N} \geq 4 \Delta \varepsilon_{e}+\Delta \varepsilon_{T}
$$

where the increment of elastic strain is given as

$$
\Delta \varepsilon_{\mathrm{e}}=\frac{\Delta \sigma_{\mathrm{T}}}{\mathrm{E}}
$$

where $\Delta \sigma$, is the stress increment between the current state and the shakedown condition (Fig. 17) and $E$ is the modulus in the transverse direction. The thermal strain is given as

$$
\Delta \varepsilon_{\mathrm{T}}=\Delta \alpha \cdot \Delta \mathrm{T}
$$

where $\Delta \alpha$ is the difference in coefficient of thermal expansion between fiber and matrix and $\Delta \mathrm{T}$ is the temperature increment between the current state and the shakedown condition. The experiments, Fig. 9, shows a ratcheting rate that is lower than the upper bound (6). 
However, the incremental change in ratcheting rate for operating conditions for outside the shakedown condition follows the upper bound.

The failure strain can differ by over an order of magnitude depending on the operating condition. This behavior which is illustrated in Fig. 11 has been observed previously by Cottrell [1964]. It is known for Al-Li [Pilling and Rindly, 1986] that ductile failure is the result of void nucleation and growth from small particles. In the studies of Hancock and Mackenzie [1976] it is suggested that when failure is the result of void nucleation and growth that the effective strain $\varepsilon_{\mathrm{f}}$ at failure for multiaxial state of stress has the form

$$
\varepsilon_{\mathrm{f}}=1.65 \cdot \varepsilon_{0} \exp \left\{-\frac{1}{2} \frac{\sigma_{\mathrm{kk}}}{\bar{\sigma}}\right\}
$$

where $\varepsilon_{0}$ is the uniaxial failure strain, $\sigma_{\mathrm{kk}}$ is the sum of the principal stresses and $\bar{\sigma}$ the effective stress. The failure strain for this matrix in uniaxial tension is reported to be approximately 0.3 [Sakui and Tamura, 1969] and Jansson [1990] has reported in tests on the composite under consideration that the strain for in-plane shear parallel to the fibers is 0.2. From the computer studies it has been determined that transverse loading alone introduces a multiaxial stress state for which $\frac{\sigma_{\mathrm{kk}}}{\bar{\sigma}}=6.0$ over a large region of the matrix at fracture. This is not greatly different from the values present in the classical Prandtl punch problem. For a history dependent stress state the damage equation of Eq. (9) is equivalent to

$$
\int_{0}^{\bar{\varepsilon}_{\mathrm{f}}} \exp \left(\frac{\sigma_{\mathrm{kk}}}{2 \bar{\sigma}}\right) \mathrm{d} \bar{\varepsilon}=1,65 \varepsilon_{0}
$$

Applying the formula (10) for the failure strain and using the data in Figs. 19 and 20 gives the failure strain for transverse loading

$$
\varepsilon_{11}^{\mathrm{f}}=0.9 \%
$$


which compares quite well with the observed failure strain of $0.8 \%$ in the transverse direction. Applying (10) for the thermomechanical loading histories gives the predicted failure strain is shown in Fig. 11. The observed failure strain is higher than the predicted by the model for low transverse stress. However, the model gives the right trend but clearly requires modification.

In the model it is assumed that catastrophic failure coincides with the condition when local failure occurs. This gives an accurate prediction when the transverse load is close to the limit load when a small defect is sufficient to trigger the failure.

In the case of low transverse loading the loss of load carrying capacity occurs in a small volume of matrix material and may not be sufficient to cause global fracture. The damage has to be extended over a larger volume and the calculations give the strain for the first matrix failure and not the strain for which the damage causes global instability. This explanation can explain the observed difference in failure strains for low and high transverse loading. The analysis required to illustrate this failure mechanism would require calculations which follow the growth of damage throughout the matrix and it has not yet been attempted.

\section{CONCLUSIONS}

When the metal matrix composite was subjected to a constant transverse stress and cyclic temperature it is found that after an initial transient response the material reaches a steady state condition.

For loading conditions which fall within a shakedown condition the increment of strain over a cycle is zero. However, if the shakedown condition is exceeded there is an increment of irreversible strain after each cycle of temperature. The shakedown condition is defined by the relation 


$$
\mathrm{g}\left(\sigma_{\mathrm{T}}, \Delta \mathrm{T}\right)=\frac{\sigma_{\mathrm{T}}}{\sigma_{\mathrm{s}}}+\frac{\Delta \mathrm{T}}{\Delta \mathrm{T}}-1=0
$$

where $\sigma_{\mathrm{s}}=1.3 \sigma_{\mathrm{y}}$ and $\Delta \mathrm{T}_{\mathrm{s}}$ is defined by

$$
\frac{E_{m} \Delta \alpha \Delta T_{s}}{\sigma_{y}}=1.4
$$

Predictions of finite element computations agree reasonable well with the experimental observations of the shakedown surface. The differences exist presumably because of the deficiencies in the constitutive equations of the matrix which in the calculations are assumed to be of a very simple form.

The increment of strain per cycle is also found to be a function of the function which defines the shakedown surface so that

$$
\frac{\mathrm{d} \varepsilon_{1}}{\mathrm{dN}}=\exp \left(9.5 \cdot 10^{-2} \beta\right)-1
$$

where $\beta$ is defined as

$$
\beta=g\left(\sigma_{\mathrm{T}}, \Delta \mathrm{T}\right)
$$

The transverse failure strain varies substantially with operating condition. The failure strain is $0.08 \%$ when transverse stress is the only loading, and it increases to $12 \%$ when the transverse stress is $30 \mathrm{MPa}$ and the thermal loading is sufficiently high to cause ratcheting. 


\section{Acknowledgements}

The work was supported by a grant from the NASA-Lewis Research Center. The authors wish to express their gratitude to Dr. Peter Sotkovszki for his help with the metallurgical studies and to Chrysler Corporation for supplying the composite material. 


\section{Appendix: Determination of Shakedown Surface and Ratchet Strains}

A simple calculation has been performed which provides physical insight and some limited quantitative information.

The composite consists of an elastic perfectly plastic metal matrix with modulus $E_{m}$ yield stress $\sigma_{\mathrm{y}}$. A Tresca yield condition is assumed. The fiber with modulus $\mathrm{E}_{\mathrm{f}}$ is assumed to remain elastic. It is also assumed that the stresses are constant in the matrix and the fiber. This is an approximation which satisfies equilibrium and consequently shall tend to give lower bounds on stiffness, limit load and shakedown conditions.

Since the fiber modulus $E_{f}$ is five times higher than the matrix modulus $E_{M}$ the elastic matrix response is readily calculated using the condition

$$
\varepsilon_{3}=\frac{\sigma_{3}}{E_{m}}-\frac{v_{m} \sigma_{1}}{E_{m}}+\Delta \alpha \Delta T=0
$$

where $\sigma_{1}$ is the stress corresponding to the transverse loading and $\sigma_{3}$ is the stress in the fiber direction acting on the matrix. Hence

$$
\sigma_{3}=-E_{m} \Delta \alpha \Delta T_{1}+v_{m} \sigma_{1}
$$

Using the Tresca Yield Condition plastic yielding occurs when

$$
\sigma_{3}-\sigma_{1}=-\sigma_{\mathrm{y}}
$$

and eliminating $\sigma_{3}$ gives

$$
\sigma_{1}\left(1-v_{\mathrm{m}}\right)=\sigma_{\mathrm{y}}-E \Delta \alpha \Delta \mathrm{T}_{1}
$$

Plastic deformation occurs if temperature is increased by a further amount $\Delta T_{2}$. Since $\Delta \varepsilon_{3}$ $=0$ then 


$$
\Delta \varepsilon_{3}^{\mathrm{P}}+\Delta \alpha \Delta \mathrm{T}_{2}=0
$$

and from the normality rule $\Delta \varepsilon_{3}^{\mathrm{p}}=-\Delta \varepsilon_{1}^{\mathrm{p}}$ the plastic strain $\varepsilon_{1}^{\mathrm{p}}$ is given by

$$
\Delta \varepsilon_{1}^{p}=\Delta \alpha \Delta T_{2}
$$

Now when the temperature is decreased by an amount $\Delta \mathrm{T}_{3}$, elastic unloading takes place until the yield condition $\sigma_{3}-\sigma_{2}=\sigma_{y}$ is reached. Since $\sigma_{2}=0$ then

$$
\sigma_{3}=-\sigma_{y}+\sigma_{1}+E_{m} \Delta \alpha \Delta T_{3}=\sigma_{y}
$$

from which the shakẹdown condition

$$
\mathrm{E}_{\mathrm{m}} \Delta \alpha \Delta \mathrm{T}_{3} \leq 2 \sigma_{\mathrm{y}}-\sigma_{1}
$$

can be deduced.

If the shakedown condition is exceeded by applying an additional temperature decrease $\Delta T_{4}$ then plastic increments of deformation occur

$$
\Delta \varepsilon_{3}^{p}=\Delta \alpha \Delta \mathrm{T}_{4}
$$

and normality gives

$$
\Delta \varepsilon_{3}^{\mathrm{p}}=-\Delta \varepsilon_{2}^{\mathrm{p}}
$$

In continued cycling no incremental accumulation of strain can occur in the third direction because the fiber is elastic. For steady state conditions is

$$
\Delta \mathrm{T}_{2}=-\Delta \mathrm{T}_{4}
$$


Let the total temperature difference be $\Delta \mathrm{T}$ for steady state conditions. Hence,

$$
\Delta \mathrm{T}=\Delta \mathrm{T}_{2}+\Delta \mathrm{T}_{3}
$$

where $\Delta T_{3}$ is the value for shakedown. The expression for the ratchet strain increment in the 1 direction is then

$$
\frac{\Delta \varepsilon_{p}^{l}}{\left(\sigma_{y} / E_{m}\right)}=\frac{E_{m}}{\sigma_{y}} \Delta \alpha \Delta T+\frac{\sigma_{1}}{\sigma_{y}}-2
$$

This increment can also be expressed in terms of the shakedown condition. Defining the function $g$ by

$$
g_{1}\left(\Delta T, \sigma_{1}\right)=\frac{E_{\mathrm{m}} \Delta \alpha \Delta \mathrm{T}}{\sigma_{\mathrm{y}}}+\frac{\sigma_{1}}{\sigma_{\mathrm{y}}}-2
$$

gives the condition for shakedown

$$
g_{1}\left(\Delta T, \sigma_{1}\right) \leq 0
$$

The ratchet increment of strain when the shakedown condition is exceeded is given by

$$
\Delta \varepsilon_{\mathrm{p}}^{\mathrm{l}}=\frac{\sigma_{\mathrm{y}}}{\mathrm{E}_{\mathrm{m}}} \mathrm{g}_{1}\left(\Delta \mathrm{T}, \sigma_{1}\right)
$$

The same model for a $v$. Mises yield condition gives the shakedown boundary

$$
g_{2}\left(\Delta T, \sigma_{1}\right)=\frac{E_{m} \Delta \alpha \Delta T}{\sigma_{y}}-\sqrt{4-3\left(\frac{\sigma_{1}}{\sigma_{y}}\right)^{2}}
$$


with the ratchet increment of strain

$$
\Delta \varepsilon_{1}^{\mathrm{p}}=\frac{3}{4} \frac{\sigma_{\mathrm{y}}}{\mathrm{E}_{\mathrm{m}}} \frac{1}{\sqrt{\left(\frac{\sigma_{\mathrm{y}}}{\sigma_{1}}\right)^{2}-\frac{3}{4}}} \cdot \mathrm{g}_{2}\left(\Delta \mathrm{T}, \sigma_{1}\right)
$$




\section{REFERENCES}

ABAQUS, Hibbit, Karlsson and Sorensen, Inc., "Users Manual," Version 4.7, 1988.

Cottrell, A.H., "The Mechanical Properties of Matter," John Wiley, New York, 1964.

Champion, A.R., Krueger, W.H., Hartman, H.S. and Dhingra, A.K., "Fiber F.P. Reinforced Metal Matrix Composites," Proc. 2nd International Conference on Composite Materials, Toronto, Metallurgical Soc. AIME, pp. 883-904, 1978.

Hancock, J.W. and Mackenzie, A.C., "On the Mechanisms of Ductile Fracture in High Strength Steels Subject to Multiaxial States of Stress," J. Mech Physics of Solids, 24, pp. 141-169, 1976.

Jansson, S., "Homogenized Nonlinear Constitutive Properties and Local Stress Concentrations for Composites with Periodical Internal Structure," Department of Mechanical Engineering, UC Santa Barbara, 1990a.

Jansson, S., "Mechanical Characterization and Numerical Modeling of Non-Linear Deformation and Fracture of a Continuous Fiber Reinforced Metal Matrix Composite," Department of Mechanical Engineering, UC Santa Barbara, 1990b.

Pilling, J. and Ridley, N., "Role of Hydrostatic Pressure on Cavitation During Superplastic Flow d Al-Li Alloy," Aluminum-Lithium Alloys III, The Institute of Metals, London, pp. 184-190, 1986.

Ponter, A.R.S. and Cocks, A.C.F., "The Incremental Strain Growth of an ElasticPlastic Body Loaded in Excess of the Shakedown Limit," Department of Engineering, Leicester University, 1982.

Sakui, S. and Tamura, M., "Yielding Phenomena of an Al-3\% Li Alloy," Transactions of the Japan Institute of Metals, Vol. 10, pp. 343-350, 1969.

Starke, E.A., Sanders, T.H. and Palmer, J.G., "New Approaches to Alloy Development in Al-Li Systems," Journal of Metals, Vol. 33, pp. 24-32, 1981. 
Table 1. Material-Constant Used in Computations

\begin{tabular}{|c|c|c|c|c|}
\hline & $\mathrm{E}$ [GPa] & $v$ & $\alpha[1 / C]$ & $\sigma_{\mathrm{y}}[\mathrm{MPa}]$ \\
\hline Fiber & 345 & 0.26 & $8.6 \cdot 10^{-6}$ & \\
\hline Matrix & 70 & 0.32 & $24 \cdot 10^{-6}$ & 95 \\
\hline
\end{tabular}

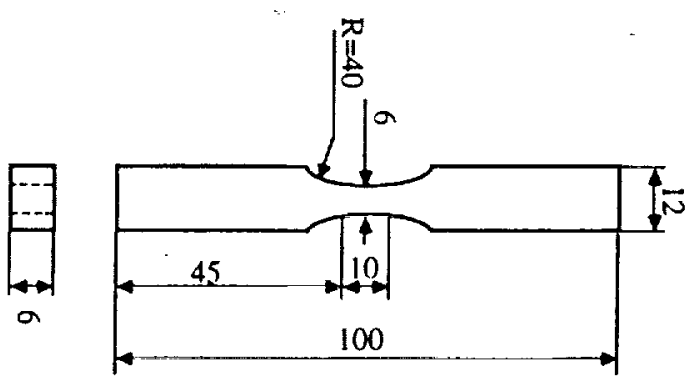

(a) Specimen Geometry.

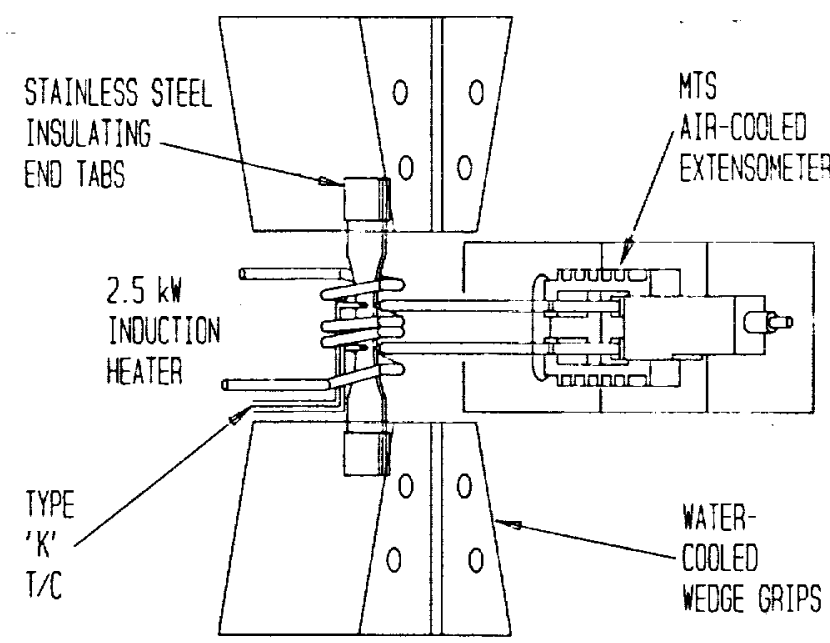

(b) Location of induction heater and MTS exiensometer.

Figure 1.-Schematic of experimental setup. 


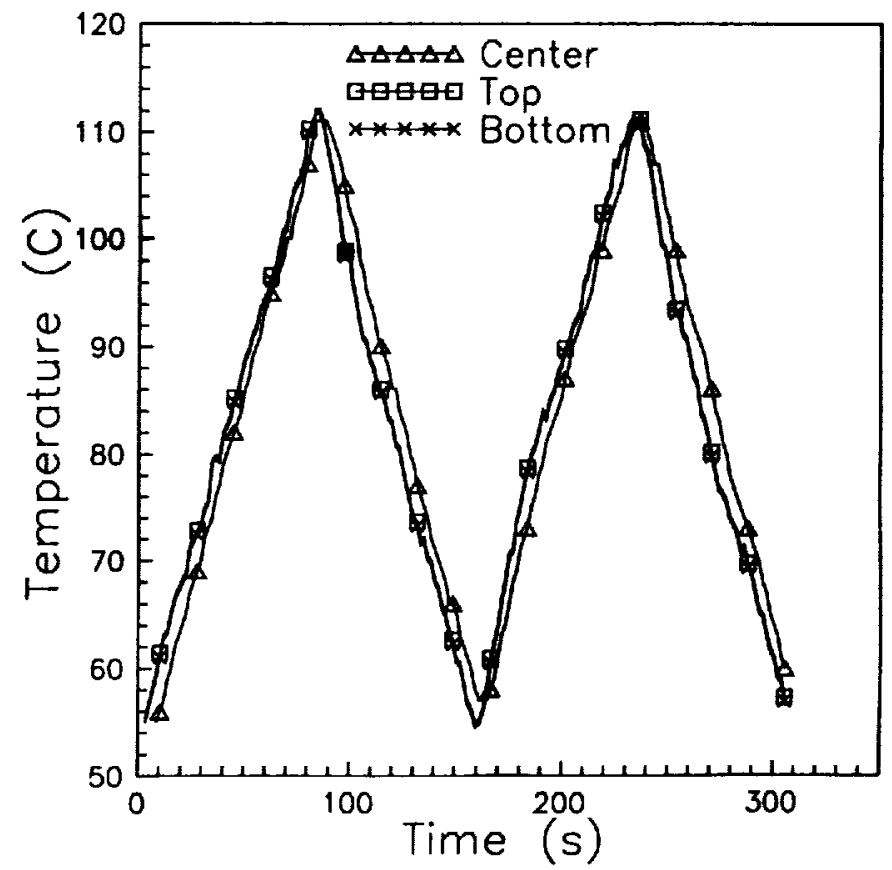

Figure 2.-Temperature distribution in specimen.

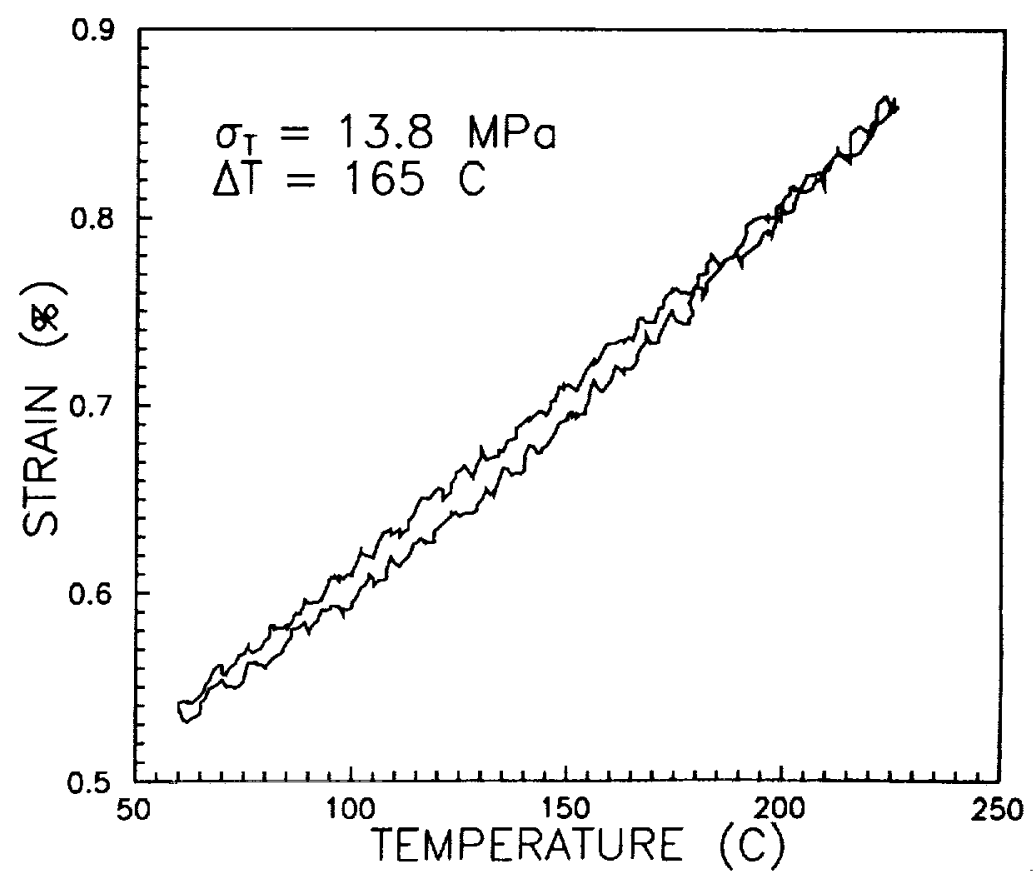

Figure 3.-Hysteresis loop. 


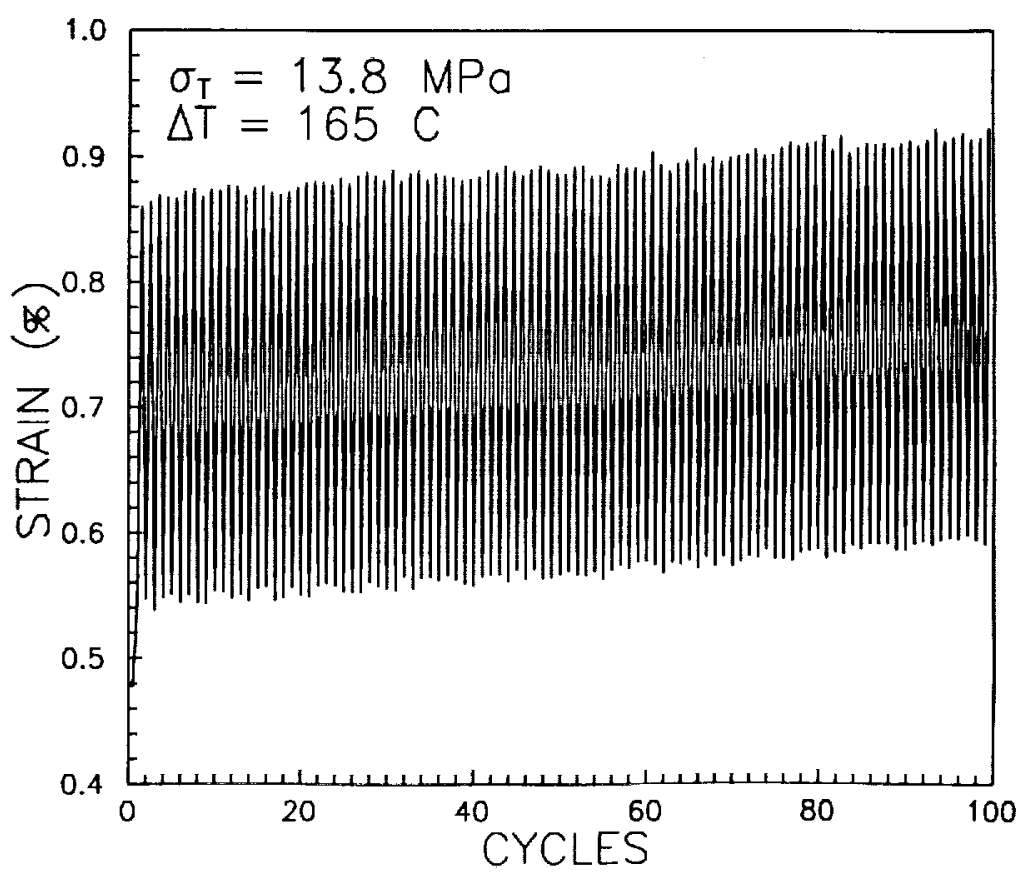

Figure 4.-Accumulation of plastic strain for a low transverse loading.

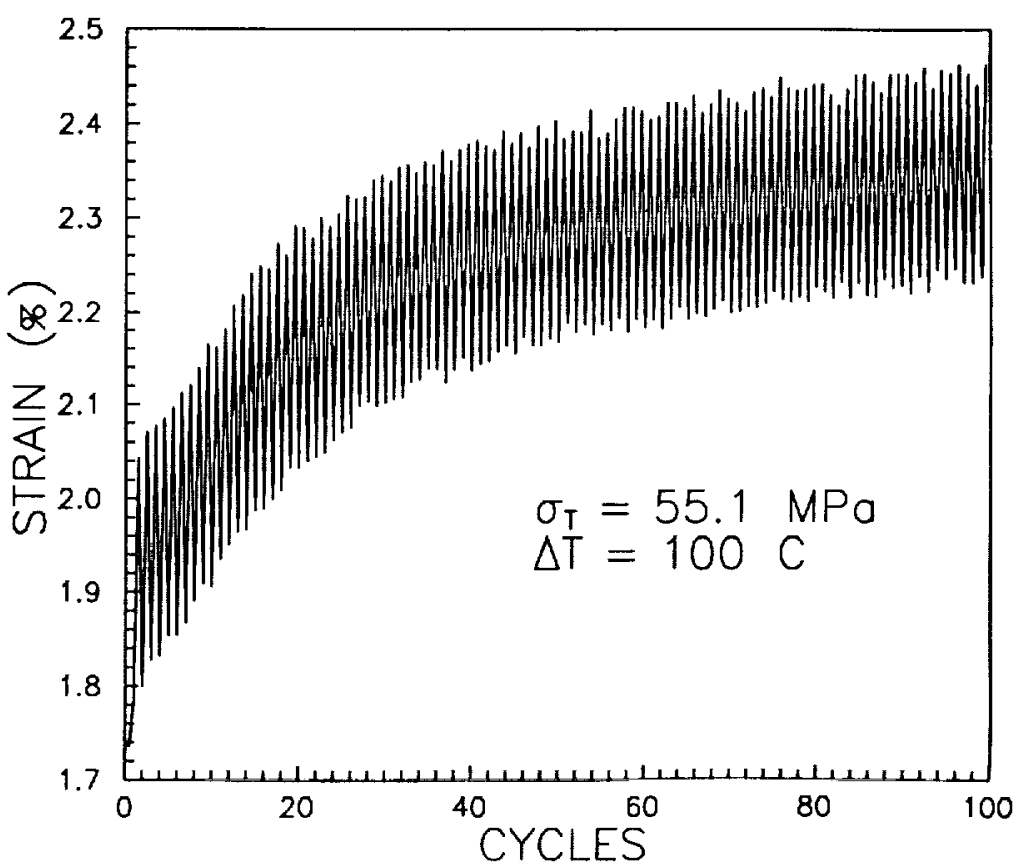

Figure 5.-Accumulation of plastic strain for high transverse loading. 


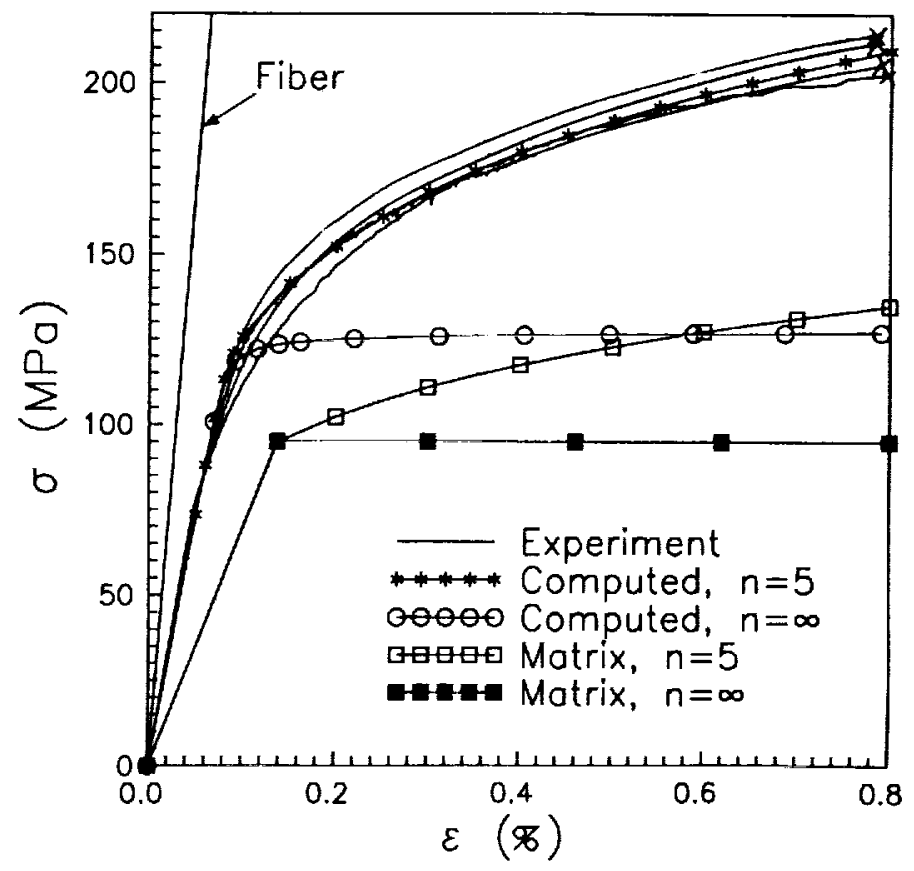

Figure 6.-Transverse stress strain curves and matrix behavior.

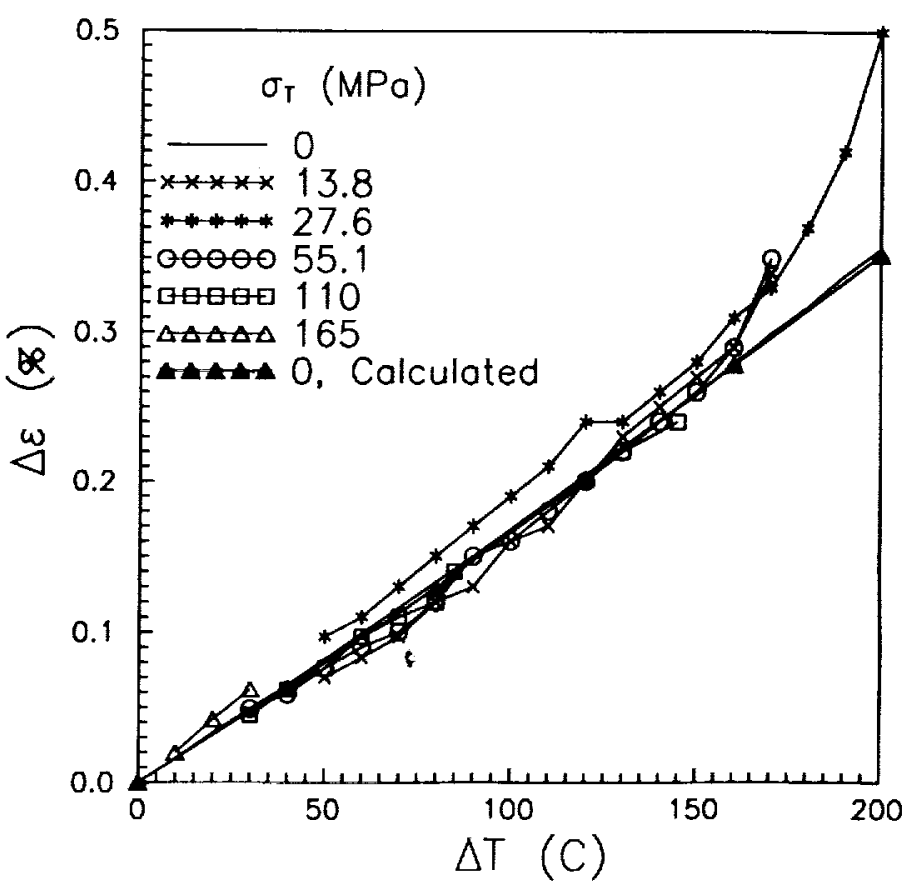

Figure 7.-Average cyclic strain as a function of transverse loading and temperature range. 


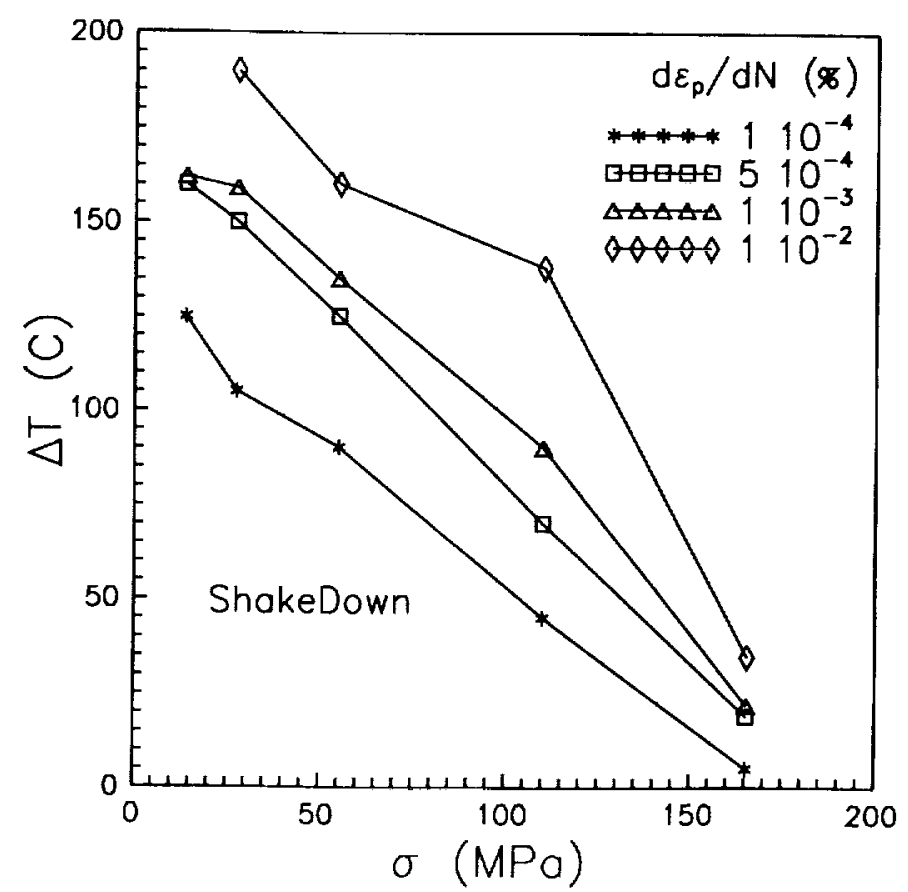

Figure 8.-Ratcheting rate as a function of transverse stress and temperature range. The rate of $10^{-4}$ is very close to the shakedown limit.

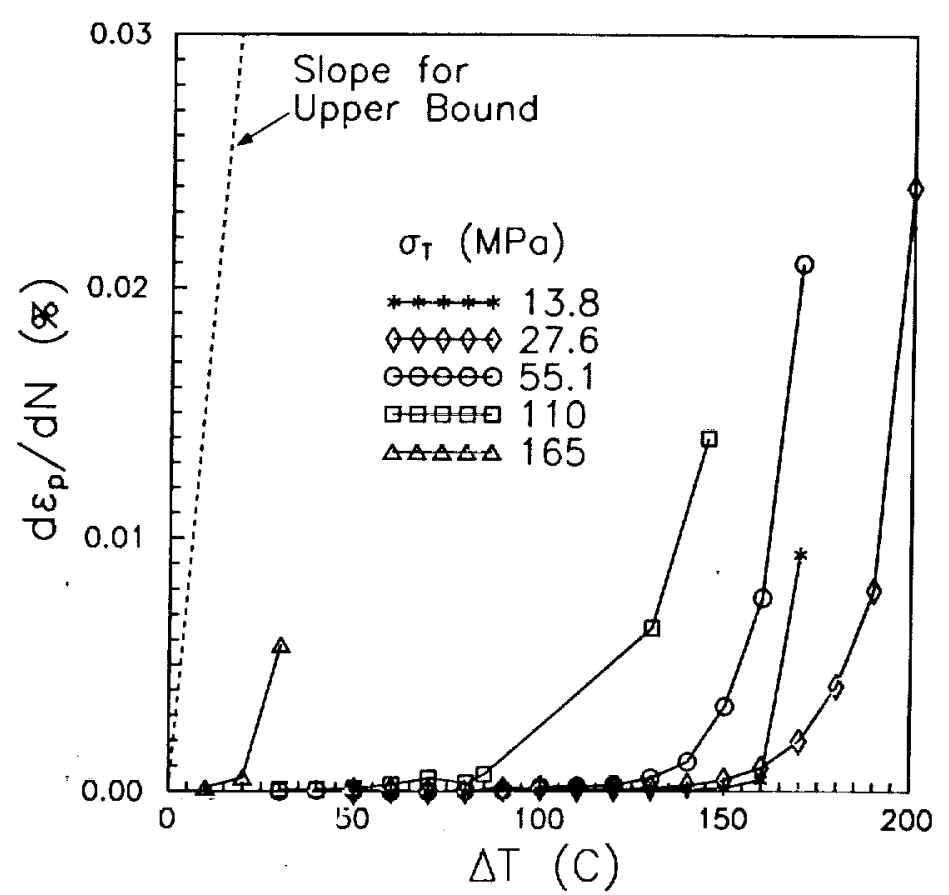

Figure 9.-Steady state racheting rate as a function of temperature and transverse stress. 


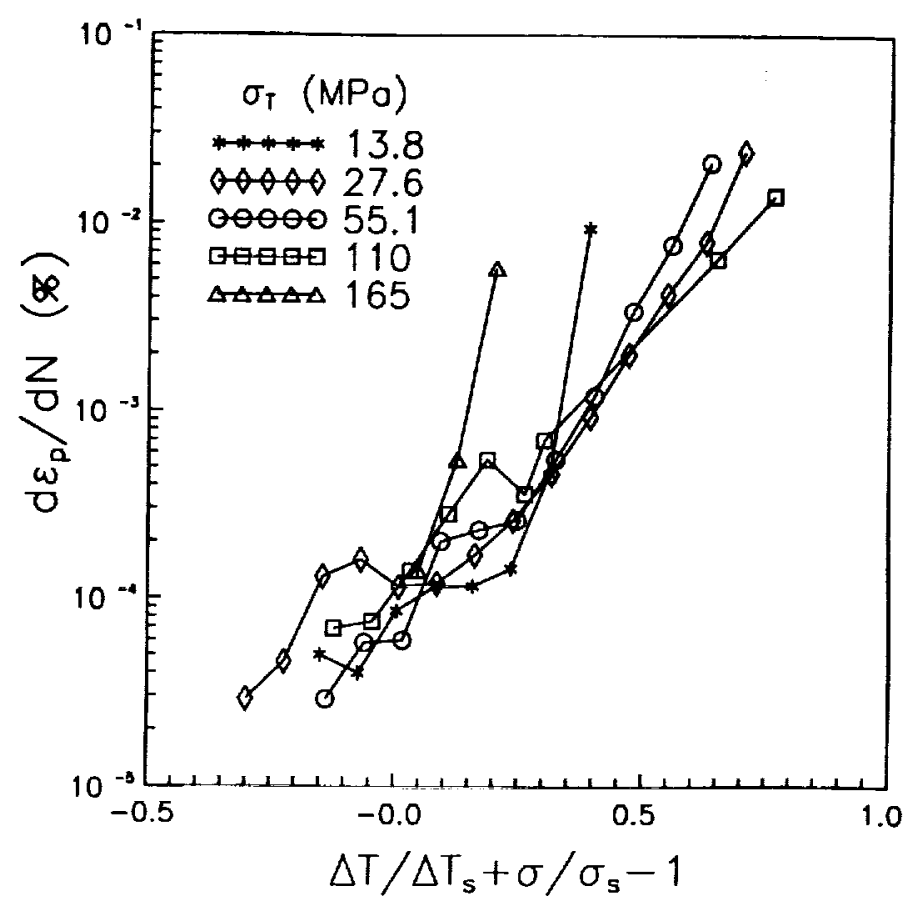

Figure 10.-Steady state ratcheting rate as a function of of the distance from the shakedown stress.

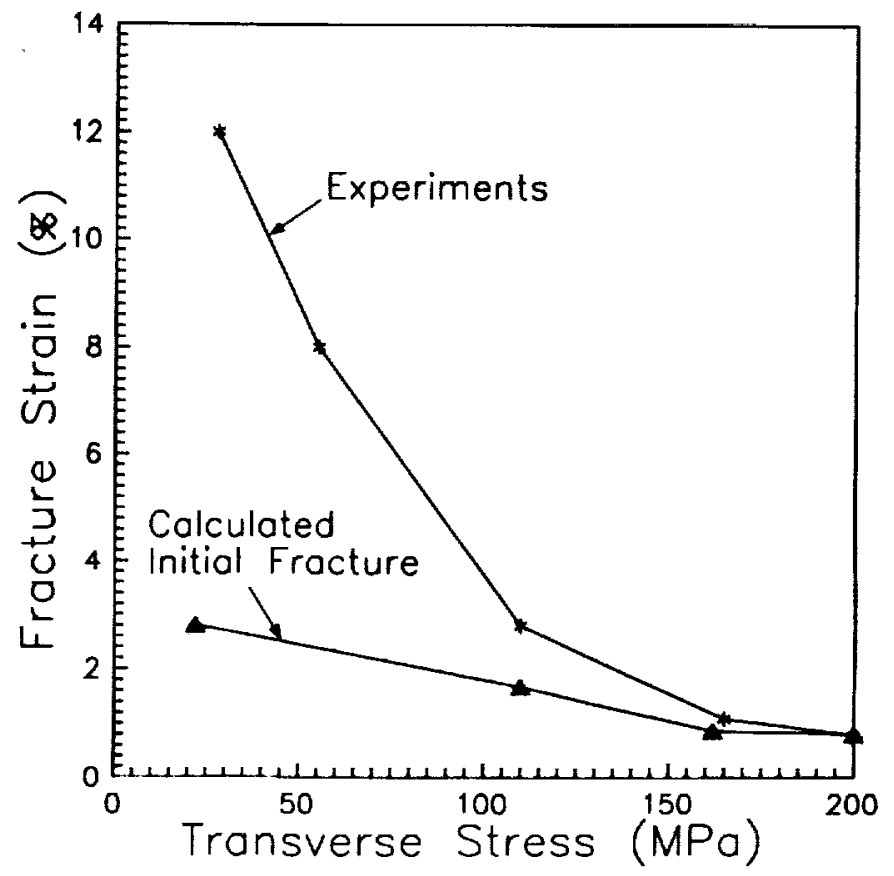

Figure 11.-Fracture strain. 


\section{ORIGINAL PAGE \\ BLACK AND WHITE PHOTOGRAPH}

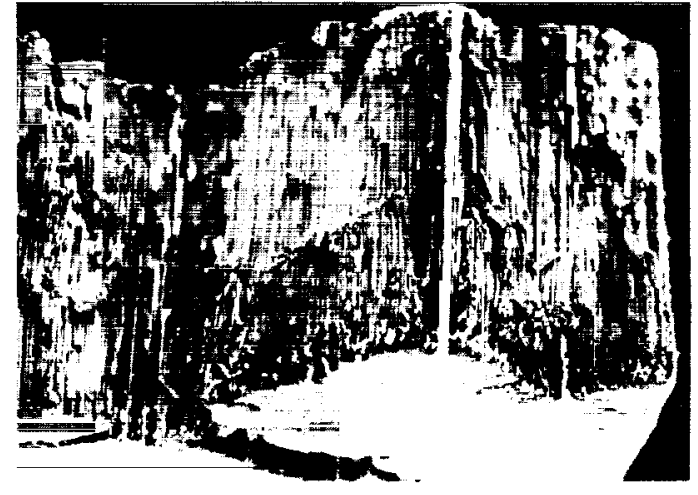

(a) A low magnification view showing the distributed cracking in the sample.

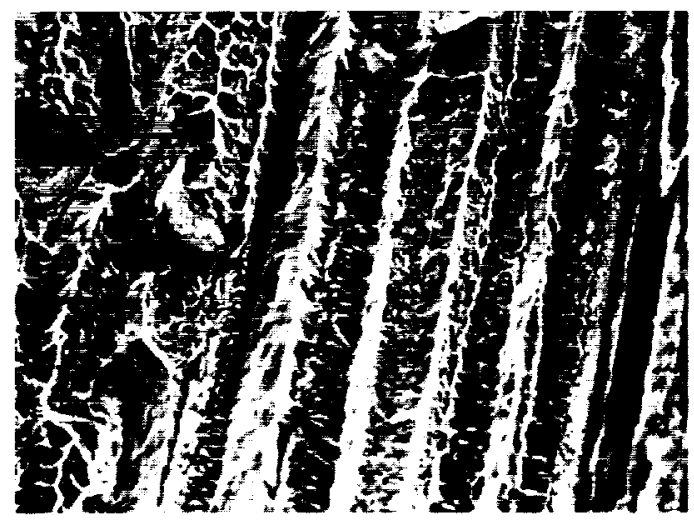

(b) A close-up indicating initial fracture close to fiber matrix interface.

Figure 12.-Typical fracture surface for low transverse loading.

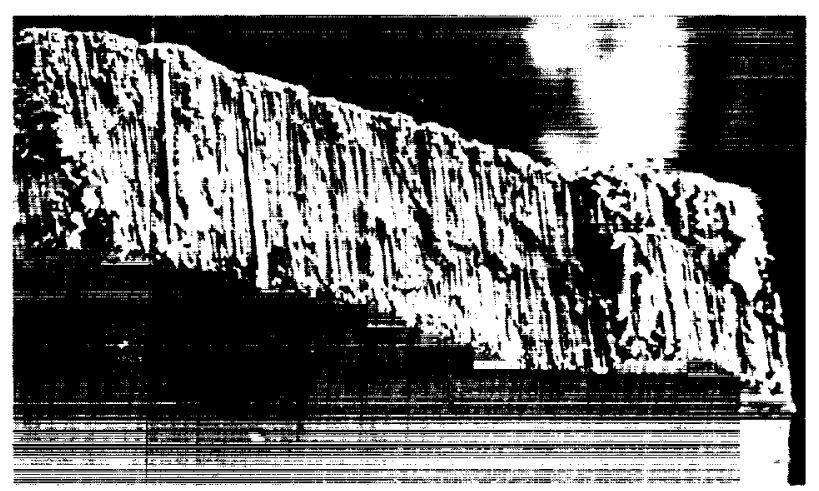

(a) A low magnification view showing that the fracture is localized to one plane.

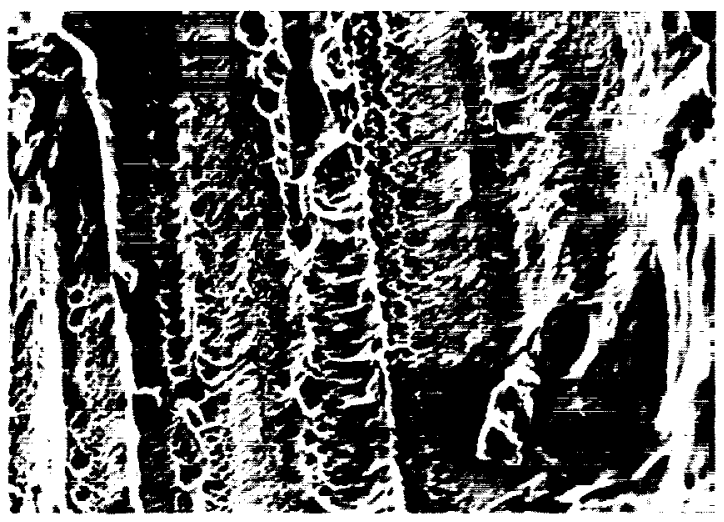

(b) A close-up showing a ductile fracture in the matrix with no traces of fiber on the fracture surface.

Figure 13.-Typical fracture surface for high transverse loading. 


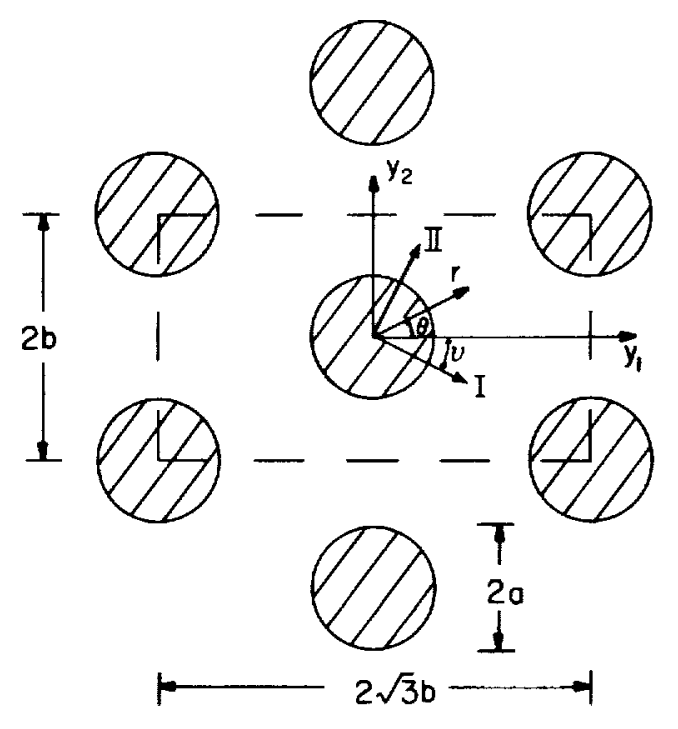

(a) Hexagonal array with unit cell indicated.

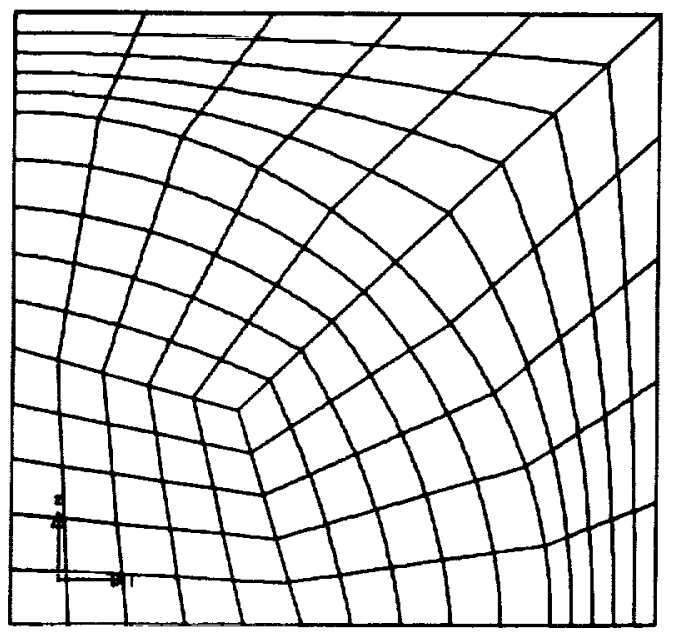

(b) Finite element mesh.

Figure 14.-Identical perodic microstructure.

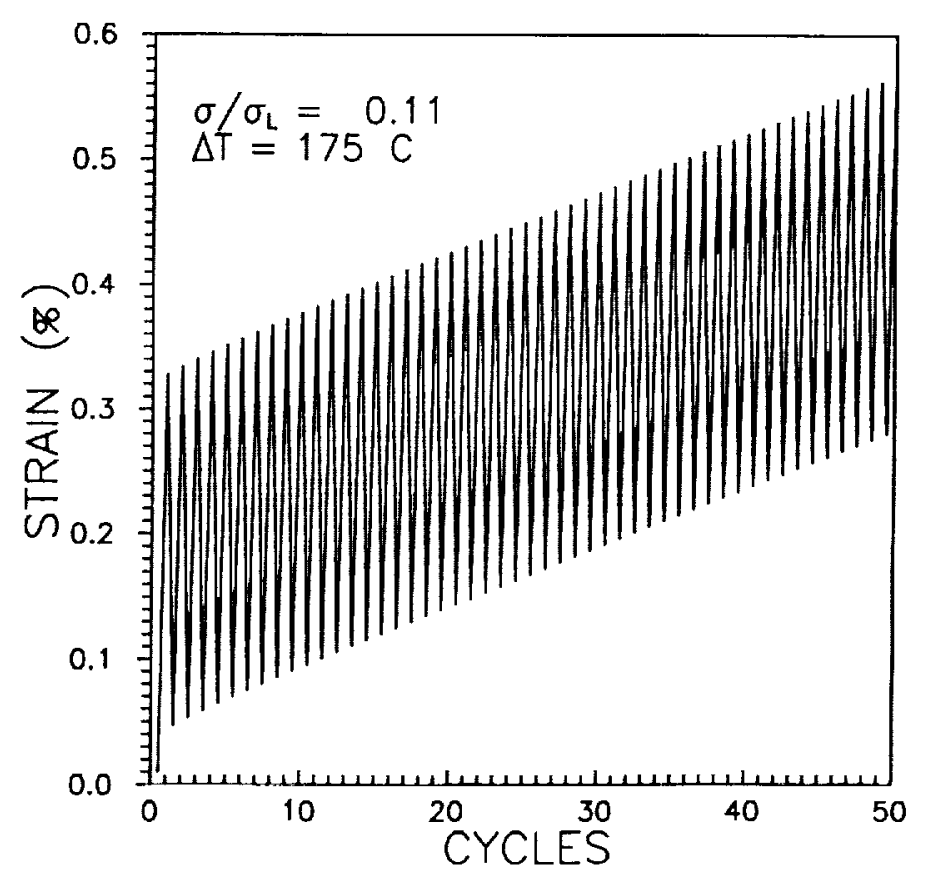

Figure 15.-Calculated strain accumulation for low transverse loading. 


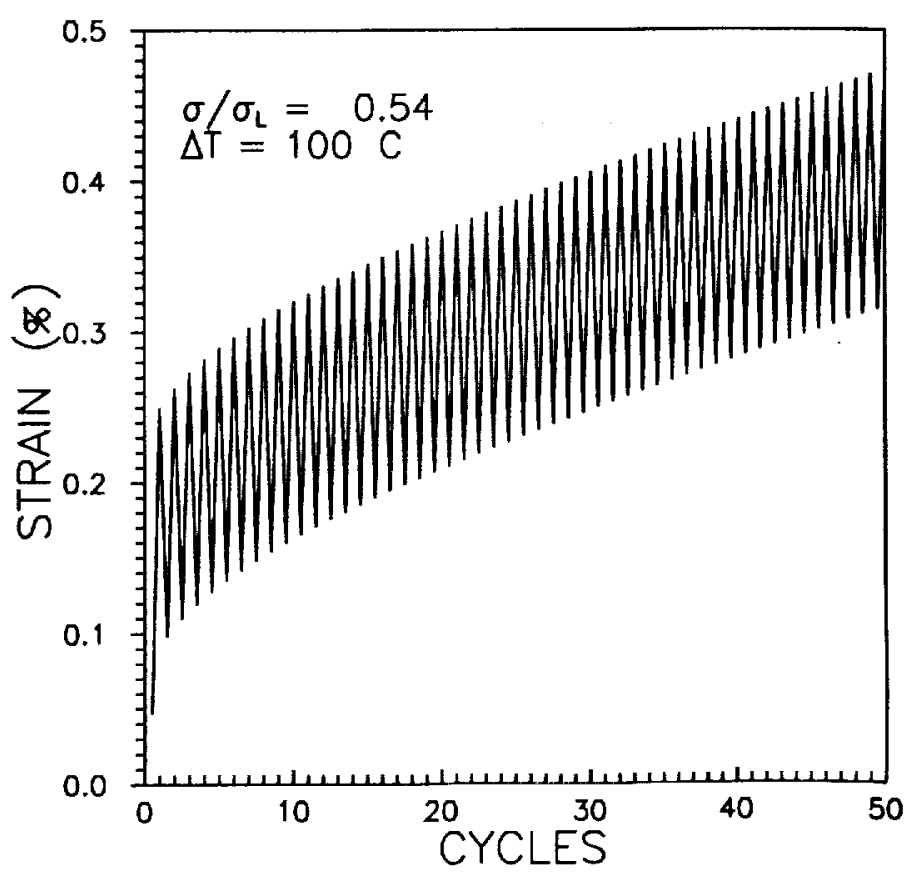

Figure 16.-Calculated strain accumulation for high transverse loading.

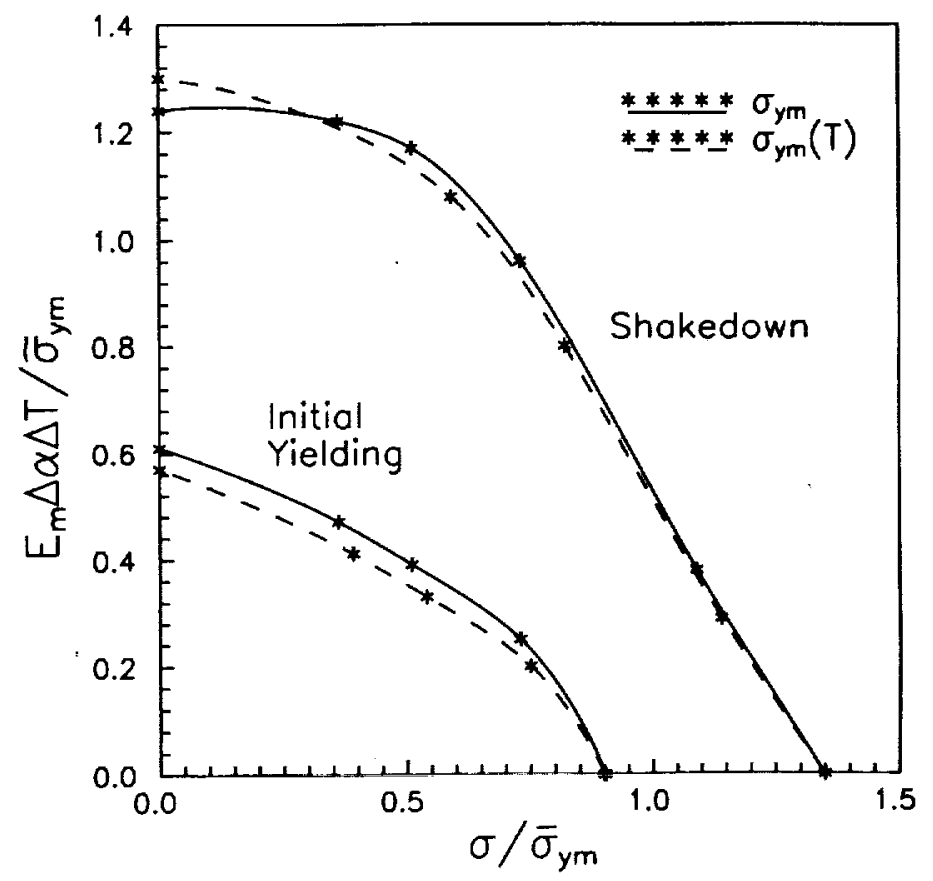

Figure 17.-Calculated initial yield surface and shakedown region for a temperature independent and exponentially temperature dependent yield stress. 


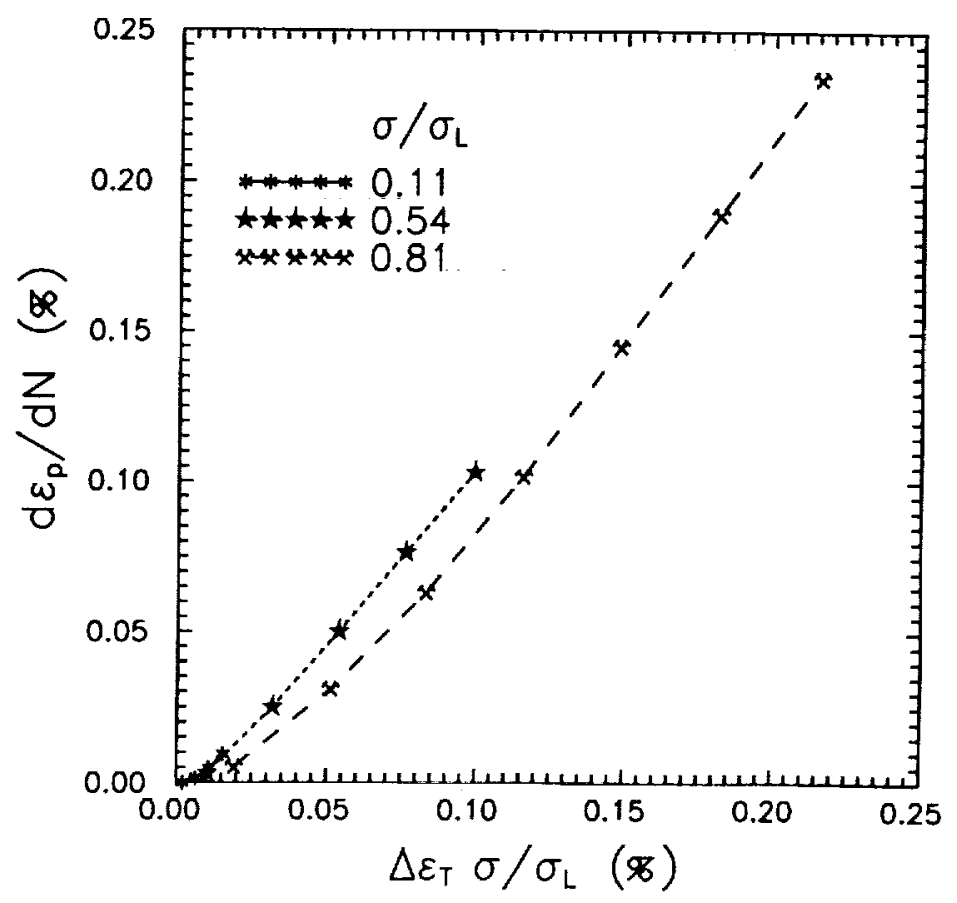

Figure 18.-Calculated steady state strain accumulation as a function of strain range and transverse loading.

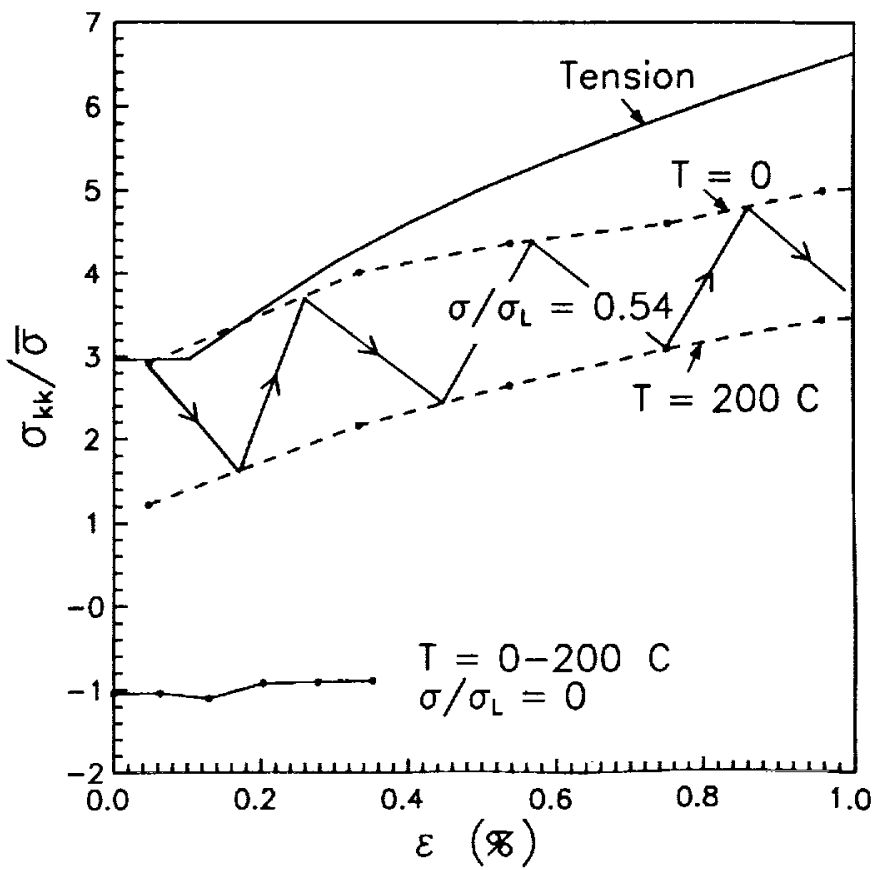

Figure 19.-Calculated maximum void growth factor in the matrix for different loadings. 


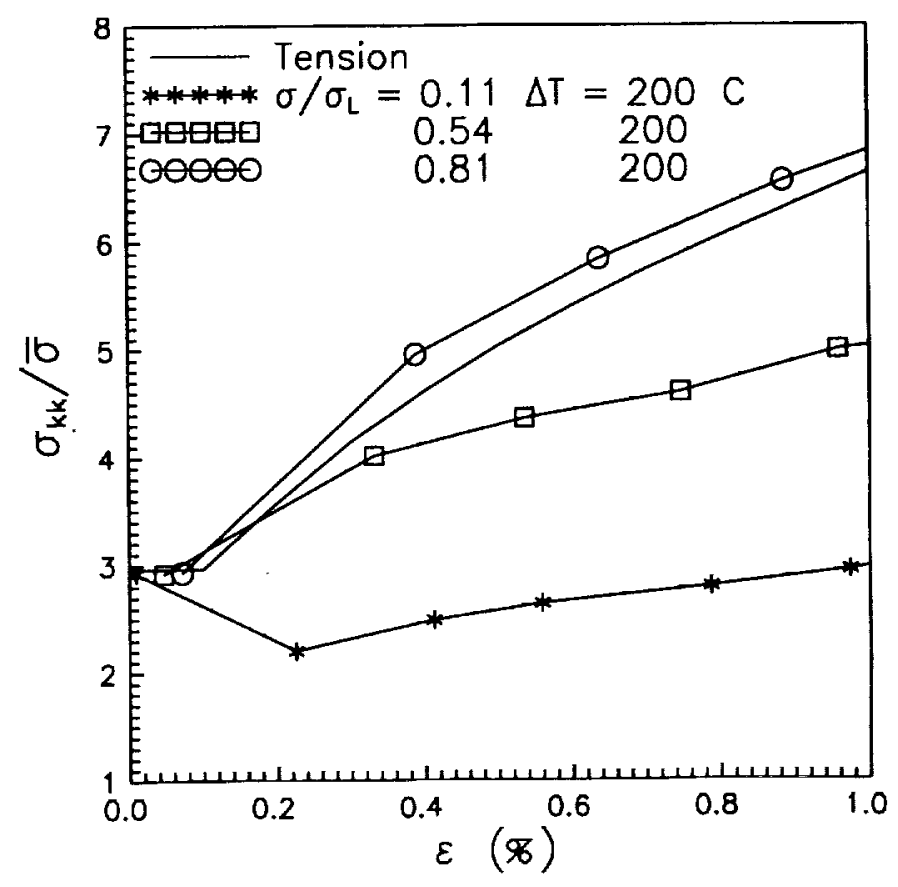

Figure 20.-Highest void growth factor in each cycle as a function of transverse strain.

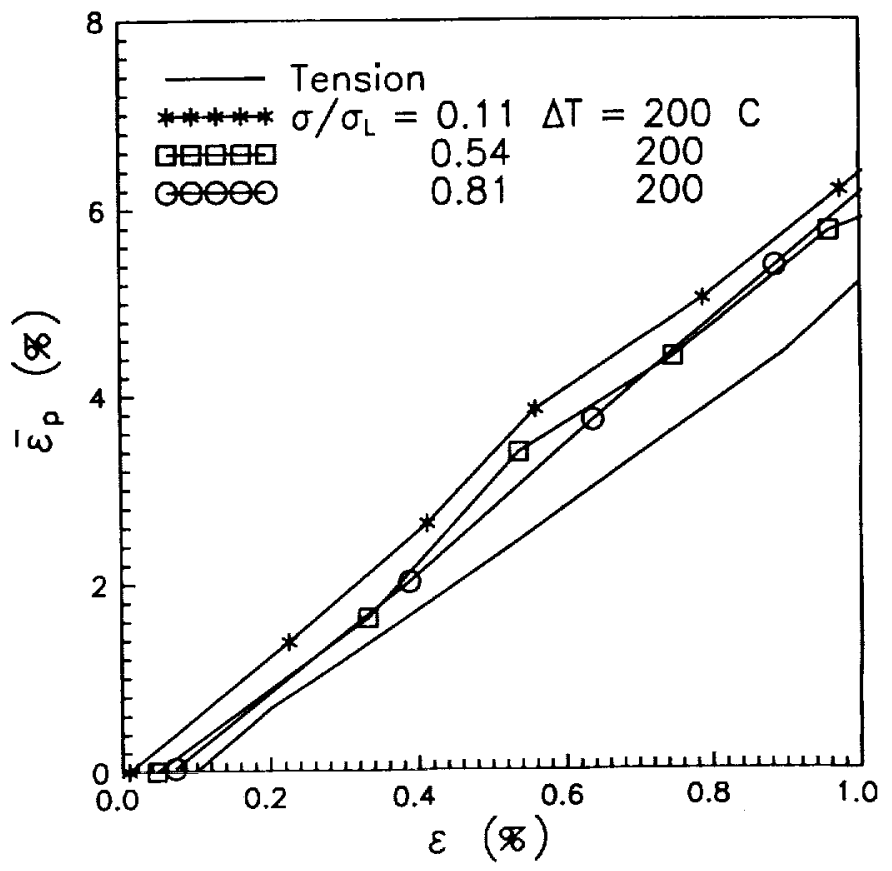

Figure 21.-Relation between effective strain at the location of the highest void growth factor and transverse strain. 


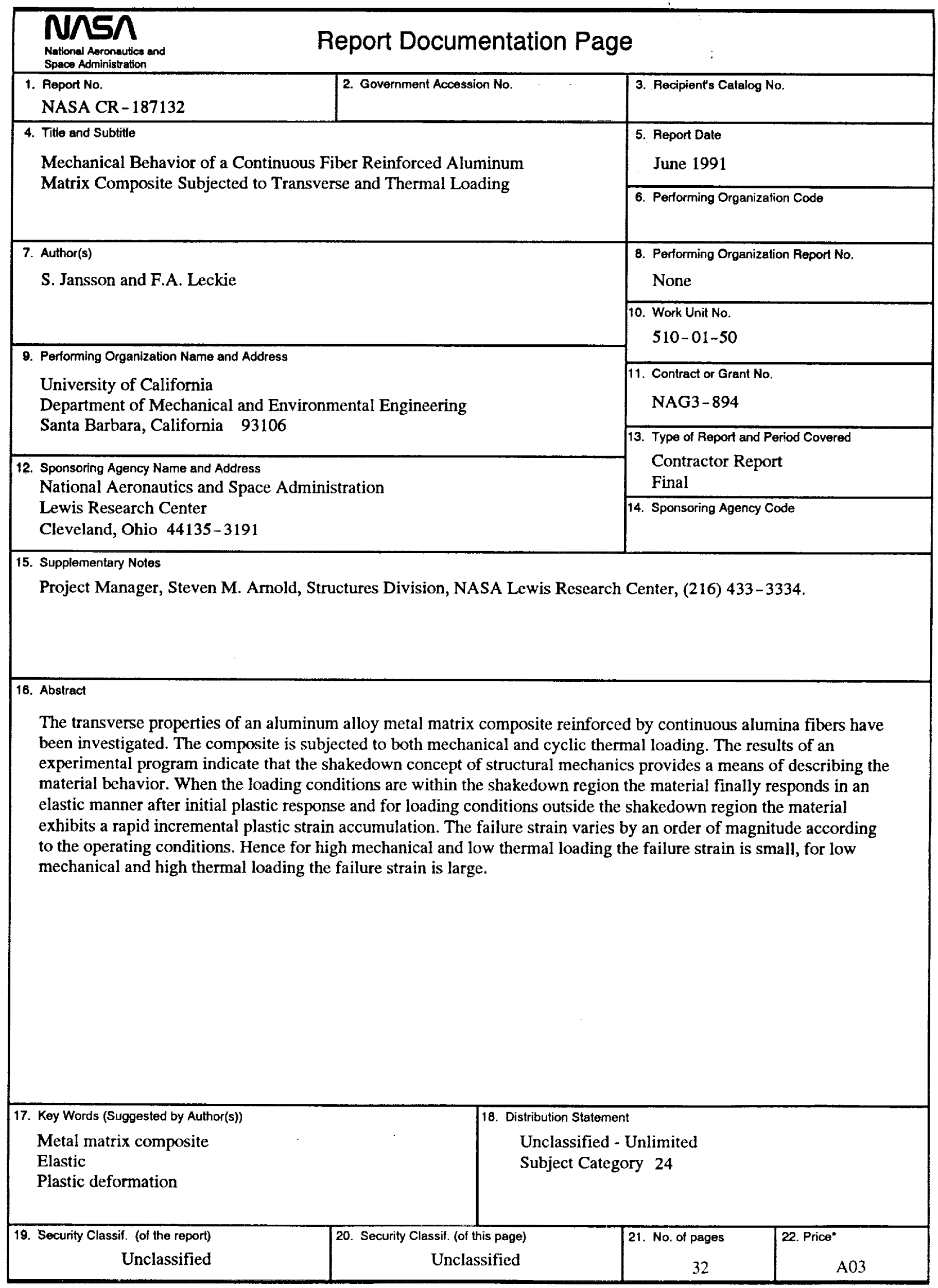

